\title{
Student Status Supervision in Ideological and Political Machine Teaching Based on Machine Learning
}

\author{
Chang $\mathrm{An}^{1 *}$ \\ ${ }^{1}$ Liaoning Finance Vocational College, Shenyang 100122, Liaoning, China
}

\begin{abstract}
Under the premise of active in the field of machine learning, this paper takes online teaching system of ideological and Political education as an example to study machine learning and machine teaching system. In order to specifically understand the current situation of the construction and application of machine teaching based on supervised teaching of ideological and political theory courses in local colleges and universities, this experiment first conducted a statistical analysis of the learning results of the surveyed classes in two semesters from March 2020 to December 2020. The experimental data show that there is a positive interaction between teachers and students. Most students use the interactive communication mode of machines, while a small number of students use real-time interactive discussions with teachers. The experimental results show that the excellent rate of $\mathrm{ABC}$ classes in the first semester is $80 \%, 82 \%$ and $90 \%$, respectively, through the machine-supervised teaching mode. Therefore, supervised machine learning can greatly help students improve their academic performance. In the future, we should further explore the application of other personalized and extensible machine learning methods in quality education.
\end{abstract}

\section{Introduction}

For a machine delivery system, the course provider and the course learner themselves cannot intervene. In order to make it easier for students to learn the knowledge they want to learn, the system should have a full understanding of the three objects of teachers, students and courses, and then carry out the service in line with users' expectations. However, traditional algorithms extract specific data based on surveys and other methods, and then use fixed algorithms to analyze users and projects $[1,2]$. However, this method may not be able to reasonably process part of the data under certain circumstances, resulting in a large proportion of some data and affecting the service quality. Moreover, algorithm design is difficult, while optimization using machine learning method can improve the accuracy of analysis and reduce the process of algorithm design [3, 4].

Learning is an important intelligent behavior possessed by human beings, but what it is has long been controversial. There is still no unified definition of machine learning. Tomasz et al. defined machine learning as "machine learning is a subject studying artificial intelligence, and artificial intelligence is its main research object, especially how to improve the performance of specific algorithms in experiential learning" [5]. Grzegorz et al. believe that machine learning is the focus of artificial intelligence and the fundamental way to make computers intelligent. It is established on the basis of cognitive discipline and so on, and learning can imitate the learning mechanism of human beings through mathematical modeling, so as to obtain the learning model oriented to specific functions, so as to autonomously learn and study the general learning theories and algorithms [6].

Machine learning is a discipline integrating computer science, probability theory, statistics, biological science, etc., which is based on computer science and aims to realize self-learning and self-improvement of computers $[7,8]$. As the foundation and core of its implementation is algorithm, its growth requirement is data, and its application result is the prediction result. The function of machine learning to predict results has made it widely used, such as data mining, computer vision, natural language processing, etc. $[9,10]$.

\section{Research on Student Status Supervision in Ideological and Political Network Teaching Based on Machine Learning}

\subsection{Research on Machine Learning Algorithms}

In the world of machine learning, there are different ways to model a real problem, depending on the type of data. The first thing to consider is the way of learning. There are three ways of machine learning. Supervised

*Corresponding author e-mail: anchang@lnfvc.edu.cn 
learning, unsupervised learning, semi-supervised learning.

(1) Supervised learning is the process of finding the corresponding output from an input data through a function generated by the existing corresponding relationship. It is usually defined as given input data $X \in$

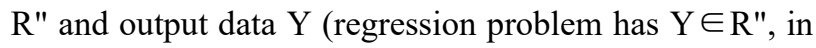
classification problem $\mathrm{Y} \in\{+1,-1\})$. Under this approach to learning, input the training sample, each group of training samples has a clear mark, by learning to establish a prediction model, and then forecast model compare to the actual situation of the training sample, and constantly adjust the prediction model, make it ultimately achieve the desired accuracy, and according to this model to predict the new conditions. Common regression classification problems adopt this learning method. The commonly used algorithms are support vector machine algorithm, Bayesian algorithm and so on.

(2) The training samples of unsupervised learning input are not clearly marked, and the learning model established by unsupervised learning is only to infer some internal structures of the sample data. Most commonly used in clustering problems, the goal is to gather samples of similar properties together, but it is not required to calculate what the similar properties between them are.

(3) Compared with supervised learning, the main difference of semi-supervised learning is that some of the input training samples are marked and some are not. The model is also used for prediction, but the model must first learn the internal structure of the sample data in order to reasonably organize the data for prediction. The regression and classification problems mentioned above can also be applied to this learning approach. Graph Inference algorithm and SVM are commonly used algorithms.

In practical applications, supervised and unsupervised learning models are the most commonly used ones.

\subsection{Supervised Learning Algorithm in Machine Learning}

Supervised learning usually means that the classifier is trained under the "supervision" of the labeled training sample. The prerequisite is to know how many classes to learn in the training sample. The accuracy and accuracy of the classifier trained by this learning method are generally better than those obtained by unsupervised learning. During the learning period, some outlier data may be incorporated into the training set data, which makes the classifier tend to overfit the data. The statistical characteristics of the flow are calculated from the original machine flow data, and the flow sampling is carried out to complete the sample acquisition. In the machine learning training stage, the feature selection is carried out first, then the supervised learning algorithm is used for training, and finally the classifier model is obtained. In classification learning, algorithms commonly used for traffic identification include naive Bayes, support vector machine, decision tree and other algorithms. The decision tree algorithm is described in detail below:

Decision tree learning is an inductive learning algorithm, it in a large number of irregular, no order sample instance as the foundation, the purity as measure, reasoned decision tree representation, get the concise classification rules, to establish a tree classifier model, to be able to classify unknown data, identification and prediction, the simplest form of decision tree is the most obvious choice only one degree of differentiation of attribute data set.

The decision tree consists of a root node, multiple internal nodes, and leaf nodes. The topmost node of the tree is the root node. Each inner node represents the child's tests on an attribute, each branch represents a test output, and each leaf node represents a class or class distribution. The first step of decision tree classification is tree building: it is to establish decision tree model with training set. This is a data, summarized the knowledge of the process, in particular, starting from the root node selection properties continuously variable of data set, the purity of data set after each divided down its biggest, until after the data set is basically the same classification, it outputs a leaf node, eventually to build a complete decision tree. The second step is classification test. For each test sample in the test set, the test starts from the root node according to the corresponding attribute of the node, and continues to test the new attribute along the branch of the decision tree until it stops splitting and reaches the leaf node. At this point, the category output by the leaf node is even though the category of the test sample belongs to.

(1) Calculate the total information entropy of sample set $\mathrm{F}$, the calculation formula is as follows:

$$
\operatorname{Entropy}(F)=\sum_{i=1}^{M} p_{k} \log _{2} p_{k}
$$

Where $\mathrm{pk}$ is the proportion of $\mathrm{Ck}$ in $\mathrm{F}$ belonging to the category.

(2) Calculate the information entropy of each attribute. Assuming that there are $\mathrm{v}$ different values on attribute $\mathrm{Fi}$, the sample set $\mathrm{F}$ can be divided into $\mathrm{V}$ subsets through attribute $\mathrm{Fi}$. If $\mathrm{Fi}$ is selected as the decision attribute, these subsets will pair with different branches of the corresponding node. The information entropy of samples divided according to FI is:

$$
\operatorname{Entropy}\left(f_{i}\right)=\sum_{\left(f_{i}\right)}\left|F_{v}\right| /|F| \operatorname{Entropy}\left(F_{v}\right)
$$

Where: Values (FI) is the set of all possible values of the attribute FI; Fv is a subset of F in which the value of attribute Fi is $\mathrm{V}$.

Calculate the information gain rate for this attribute. After dividing the sample set $\mathrm{F}$ by attribute $\mathrm{Fi}$, the information gain rate value obtained is:

$$
\operatorname{gain}\left(f_{1}\right)=\operatorname{Entropy}(F)-\operatorname{Entropy}\left(f_{1}\right)
$$

The information gain rate is: 


$$
\operatorname{gainRatio}\left(f_{i}\right) / \operatorname{splitInfo}\left(f_{i}\right)
$$

Among them:

$$
\operatorname{splitInfo}\left(f_{i}\right)=\sum_{V\left(f_{i}\right)}\left(\left|F_{v}\right| /|F|\right) \log _{2}\left(\left|F_{v}\right| /|F|\right)
$$

\section{The Experiment of Student Status Supervision in Ideological and Political Network Teaching Based on Machine Learning}

\subsection{Experimental Purpose}

In order to deeply understand the application of machine teaching in ideological and political theory course in colleges and universities, the experiment further investigated the work in a local college. Relying on a domestic MOOC platform and taking the basic principles of Marxism as the investigation object, the school has introduced machine teaching mode since the spring semester of 2020. After nearly a year of exploration, its machine teaching mode reform has achieved phased results. At present, the introduction of machine teaching mode has been extended to the four ideological and political theory courses offered by the university, with about 10,000 person-times choosing courses each year. Its exploration of machine teaching under mixed conditions has a certain typical significance.

\subsection{Experimental Methods}

Specific understanding of the local colleges as machine building and using the present situation of the teaching of ideological and political theory, this study first in March 2020 to December 2020, a total of two semesters, through online survey and data collection, the local Open University machine of ideological and political theory course construction and use of the overall situation has made the investigation and the related statistical data. Investigation project mainly includes: the local construction of ideological and political theory course teaching machine based on the main network platform, to participate in major colleges and universities, the platform of ideological and political theory courses, course has opened all the machine teaching mode, students participate in the course, course platform to create a pattern, etc.

\section{Discussion on Student Status Supervision in Ideological and Political Network Teaching Based on Machine Learning}

Table 1. Survey results of teacher-student interaction in online teaching of ideological and political theory courses in the first

\begin{tabular}{|c|c|c|c|c|c|c|c|}
\hline Course & Class & $\begin{array}{c}\text { Teacher } \\
\text { participation }\end{array}$ & $\begin{array}{c}\text { Total } \\
\text { number of } \\
\text { students }\end{array}$ & $\begin{array}{c}\text { Total } \\
\text { number of } \\
\text { questions } \\
\text { asked by } \\
\text { students }\end{array}$ & $\begin{array}{l}\text { Teachers' } \\
\text { Answers }\end{array}$ & $\begin{array}{l}\text { Percentage of } \\
\text { students } \\
\text { participating in } \\
\text { online } \\
\text { communication } \\
(\%)\end{array}$ & $\begin{array}{c}\text { Proportion } \\
\text { of } \\
\text { outstandin } \\
\text { g students } \\
(>=90 \%)\end{array}$ \\
\hline \multirow{3}{*}{$\begin{array}{l}\text { Introduction } \\
\text { to the Basic } \\
\text { Principles of } \\
\text { Marxism }\end{array}$} & A & 1 & 50 & 89 & 72 & 82 & 80 \\
\hline & B & 1 & 60 & 112 & 100 & 91 & 82 \\
\hline & $\mathrm{C}$ & 1 & 50 & 93 & 79 & 87 & 84 \\
\hline
\end{tabular}
semester

(1)A semester of interaction between teachers and students, according to the data and the positive interaction between teachers and students, and most of the students using machine learning way of interaction, a small number of students, the use of the real-time interaction with the teachers online discussion, even though this part of the students is of a smaller proportion of students to participate in the enthusiasm of the ideological and political theory class is constantly improving. At the same time, through the analysis of student achievement, we can also find that results in more than 90 points of students tend to be students who participate in online communication, introduction to basic principle of marxism, for example, in the first semester after machine of ideological and political theory teaching, the teachers and students interactive situation investigation, the data of figure 1 are shown in table 1. 


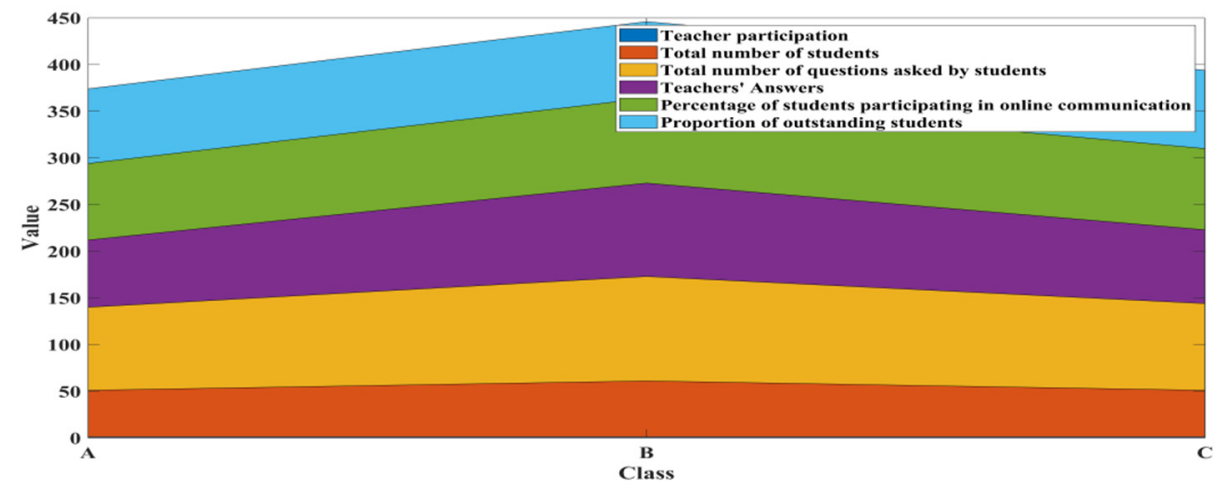

Figure 1. Survey results of teacher-student interaction in online teaching of ideological and political theory courses in the first semester

(2) According to the teaching data statistics of two semesters, we can see that the number of teachers participating in machine teaching is on the rise, and more and more teachers are participating in machine teaching. However, teachers' participation in machine teaching also has problems in the accounting of teachers' workload and performance. The workload and performance evaluation of teachers directly involved in machine teaching should be further clarified. For those who participate in classroom teaching and teachers to participate in the machine and its working performance appraisal in teaching of classroom teaching and machine also need to make further clear, ensure the work enthusiasm of the teachers, the second semester machine of ideological and political theory teaching interaction between teachers and students to investigate and found that students and teachers to machine the machine more and more teaching mode, and illustrate the machine machine teaching effect is very good.

Table 2. Survey results of teacher-student interaction in online teaching of ideological and political theory courses in the second semester

\begin{tabular}{|c|c|c|c|c|c|c|c|}
\hline Course & Class & $\begin{array}{c}\text { Teacher } \\
\text { participation }\end{array}$ & $\begin{array}{c}\text { Total } \\
\text { number of } \\
\text { students }\end{array}$ & $\begin{array}{c}\text { Total } \\
\text { number of } \\
\text { questions } \\
\text { asked by } \\
\text { students }\end{array}$ & $\begin{array}{c}\text { Teachers' } \\
\text { Answers }\end{array}$ & $\begin{array}{c}\text { Percentage of } \\
\text { students } \\
\text { participating in } \\
\text { online } \\
\text { communication } \\
(\%)\end{array}$ & $\begin{array}{c}\text { Proportion of } \\
\text { outstanding } \\
\text { students } \\
(>=90 \%)\end{array}$ \\
\hline \multirow{2}{*}{$\begin{array}{c}\text { Introduction } \\
\text { to the Basic } \\
\text { Principles of } \\
\text { Marxism }\end{array}$} & $\mathrm{A}$ & 3 & 50 & 113 & 102 & 85 & 84 \\
\cline { 2 - 8 } & $\mathrm{C}$ & 3 & 60 & 134 & 110 & 92 & 88 \\
\hline
\end{tabular}

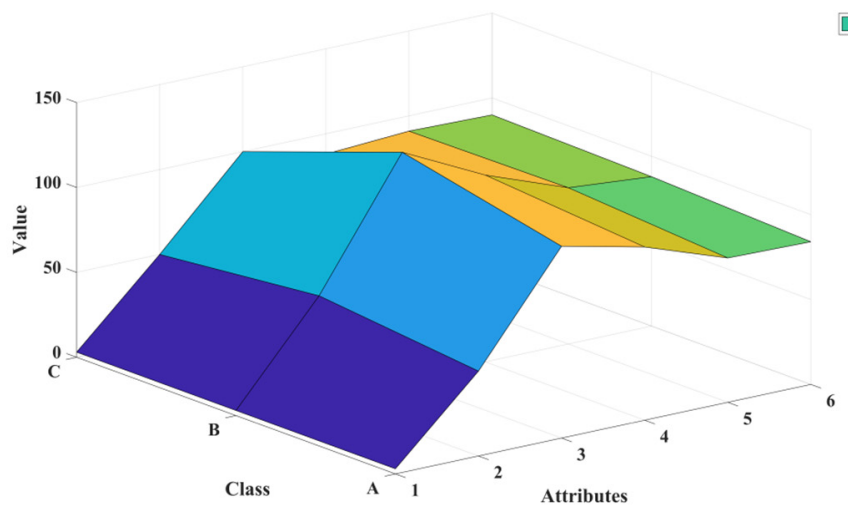

Figure 2. Survey results of teacher-student interaction in online teaching of ideological and political theory courses in the second semester 


\section{Conclusion}

Supervised learning refers to learning the information contained in marked data, classifying the unmarked data after the classification model is obtained, while unsupervised learning refers to learning the information of unmarked data directly. It takes a certain amount of human and material resources to get the labeled data, so it is usually difficult to get a large amount of labeled data. In this experiment, the machine survey learning results of $\mathrm{A}, \mathrm{B}$ and $\mathrm{C}$ classes were counted. The experimental results show that the excellent rate of $\mathrm{ABC}$ classes in the first semester is $80 \%, 82 \%$ and $90 \%$, respectively, through the machine-supervised teaching mode. Supervised machine teaching is very helpful to students' academic performance as well as the interaction between teachers and students.

\section{References}

1. Louie A K, Balon R, Beresin E V, et al. Teaching to See Behaviors-Using Machine Learning. Academic Psychiatry, 2017, 41(5):1-6.

2. Sulmont E, Patitsas E, Cooperstock J R. What Is Hard about Teaching Machine Learning to Non-Majors? Insights from Classifying Instructors' Learning Goals. ACM Transactions on Computing Education, 2019, 19(4):1-16.

3. Benato L, Connor P L S , Kasieczka G, et al. Teaching machine learning with an application in collider particle physics. Journal of Instrumentation, 2020, 15(09):C09011.

4. Martinez-Tenor A, Cruz-Martin A, Fernandez-Madrigal J A. Teaching machine learning in robotics interactively: the case of reinforcement learning with Lego ${ }^{\circledR}$ Mindstorms. Interactive Learning Environments, 2019, 27(1-4):293-306.

5. Kumar D, Mandal T. Multi-Objective Virtual Machine Placement using Improved Teaching Learning Based Optimization in Cloud Data Centers. International Journal of Applied Engineering Research, 2017, 12(21):10809-10815.

6. Tomasz R, Grzegorz K, Edward K. A Non-Destructive System Based on Electrical Tomography and Machine Learning to Analyze the Moisture of Buildings. Sensors, 2018, 18(7):2285-.

7. Jiang J, Yu L, Jiang J, et al. Angel: a new large-scale machine learning system. National Science Review, 2017, v.5 (02):102-122.

8. Li P, Li T, Ye H, et al. Privacy-preserving machine learning with multiple data providers. Future Generation Computer Systems, 2018, 87(OCT.): 341-350.

9. Ward L, Liu R, Krishna A, et al. Including crystal structure attributes in machine learning models of formation energies via Voronoi tessellations. Physical review. B, Condensed Matter and Materals Physics, 2017, 96(2):024104.1-024104.12.
10. Pathak J, Wikner A, Fussell R, et al. Hybrid Forecasting of Chaotic Processes: Using Machine Learning in Conjunction with a Knowledge-Based Model. Chaos, 2018, 28(4):041101. 